\title{
Typical Local Measurements in Generalized Probabilistic Theories: Emergence of Quantum Bipartite Correlations
}

\author{
Matthias Kleinmann, ${ }^{1, *}$ Tobias J. Osborne, ${ }^{2, \dagger}$ Volkher B. Scholz, ${ }^{3,2, *}$ and Albert H. Werner ${ }^{2, \S}$ \\ ${ }^{1}$ Naturwissenschaftlich-Technische Fakultät, Universität Siegen, Walter-Flex-Straße 3, 57068 Siegen, Germany \\ ${ }^{2}$ Institut für Theoretische Physik, Leibniz Universität Hannover, Appelstraße 2, 30167 Hannover, Germany \\ ${ }^{3}$ Institut für Theoretische Physik, ETH Zurich, Wolfgang-Pauli-Strasse 27, 8093 Zurich, Switzerland
}

(Received 22 May 2012; published 23 January 2013)

\begin{abstract}
What singles out quantum mechanics as the fundamental theory of nature? Here we study local measurements in generalized probabilistic theories (GPTs) and investigate how observational limitations affect the production of correlations. We find that if only a subset of typical local measurements can be made then all the bipartite correlations produced in a GPT can be simulated to a high degree of accuracy by quantum mechanics. Our result makes use of a generalization of Dvoretzky's theorem for GPTs. The tripartite correlations can go beyond those exhibited by quantum mechanics, however.
\end{abstract}

Introduction.-The continued success of quantum mechanics $(\mathrm{QM})$ strongly implies that it is the fundamental description of nature. However, it could still be that QM is simply a very good effective theory which breaks down if we are able to perform experiments with sufficiently high energy and precision. In this case QM would need to be replaced by a more general "postquantum" theory. In particular generalized probabilistic theories (GPTs) [1-5] have received considerable attention recently, both as a foil to better understand the features of QM, and as a powerful abstract way to reason about correlations and locality. These investigations have lead to many interesting results, including simplified and improved cryptographic schemes and primitives $[6,7]$.

If nature is actually described by a theory other than QM then the natural question arises: Why is QM such a good effective theory? A natural answer, which we investigate here, is that experimental imperfections prevent us from observing any postquantum phenomena.

Suppose that nature is described by a GPT with a highdimensional state space and corresponding high-dimensional set of all possible measurements. Observational limitations, such as detector resolution, mean that it is impossible to access most of these theoretically possible measurements. If physically implementable measurements are those chosen from some typical subset (a precise definition is given in the sequel) then we show that the bipartite correlations arising in any experiment can be modeled, to a high degree of precision, by those of QM. Note that the tripartite and multipartite correlations could go beyond those exhibited by QM: a sufficiently refined experiment involving three or more particles could exhibit behavior going beyond that possible within QM.

It is interesting to contrast our setting with that of decoherence, which models the passage from the microscopic to the macroscopic classical world [8,9]. The crucial difference here is that decoherence arises from the correlations developed between a given particle and many other inaccessible particles (in the GPT framework it is rather likely that decoherence will always lead to an effective classical theory). By way of contrast, we consider only a few particles in isolation: roughly speaking, we study the case where only the "local dimensions" are effectively truncated.

Our argument builds on several important prior ideas. The first arises from the search [10-13] for an axiomatic derivation of QM: it was realized that a reasonable physical theory should allow for the convex combination of different possible measurements, and hence the underlying sets of both states and measurements should be dual convex bodies. These developments have lead to the identification of generalized probabilistic theories as a general framework to study theories of physics going beyond QM.

The second cornerstone of our argument is the concentration-of-measure phenomenon [14,15] epitomized by Dvoretzky's theorem which states, roughly, that a random low-dimensional section of a high-dimensional convex body looks approximately spherical. This powerful result has already found myriad applications in quantum information theory, e.g., in quantum Shannon theory $[16,17]$, and quantum computational complexity theory $[18,19]$. Here we adapt the "tangible" version of Dvoretzky's theorem for our purposes.

The final idea we exploit is the observation that spherical state spaces can be simulated by sections of quantum mechanical state spaces [20]. As will become evident, our approach owes much to the recent work $[21,22]$ showing that bipartite correlations may be modeled by QM when the constituents locally obey QM.

Main result.-If the local measurements in a GPT are chosen from a typical section of the convex body of all possible measurements then, with a high degree of accuracy, they do not yield any postquantum prediction for the bipartite scenario. 
More specifically, we require that the physically implementable measurements are in essence given by the section of the convex body of all measurements with a lowdimensional $O(n)$-typical subspace. This means that the accessible measurements span a subspace and the choice of this subspace is not particular among all other subspaces of the same dimension. This is a core assumption in our argument. Although we restrict our attention here to the case of $O(n)$-typical subspaces, it is likely that our result extends to a much wider variety of typicality notions.

Our argument then implies that for most measurements given by low-dimensional subspaces the outcomes can be explained using quantum mechanics. Hence we argue that those measurement devices revealing any postquantum behavior are extremely difficult to buildsince the choice of the right subspace requires extreme fine tuning.

Probabilistic physical theories, ordered vector spaces.It is useful to formulate GPTs in the mathematical language of ordered vector spaces [2,23,24]: we begin with the description of the single-party state space and local measurements. The system is always assumed to be in a state $\omega$, which encodes the probabilities of each outcome of all the possible measurements that may be performed. The set of all possible states, state space, is denoted $\Omega$. Since any probabilistic combination of states is, in principle, preparable, $\Omega$ is a convex set. We always assume that $\Omega$ is represented as a subset of $\mathbb{R}^{n}$.

A state $\omega \in \Omega$ assigns a probability to each outcome of any possible measurement; a measurement outcome is represented by a map $f: \Omega \rightarrow[0,1]$. This map respects probabilistic mixtures of states, meaning that $f\left(p \omega_{1}+\right.$ $\left.(1-p) \omega_{2}\right)=p f\left(\omega_{1}\right)+(1-p) f\left(\omega_{2}\right)$. Extending each map linearly allows us to conclude that measurement outcomes are elements of the dual space $V$ to $\mathbb{R}^{n}$. Any such $f$ is called an effect. A special effect is the unit effect $e$ defined by $e(\omega)=1$ for all $\omega \in \Omega$. The unit effect represents a measurement with a single outcome: this is certain to occur regardless of what the state is. Convex combinations of effects are themselves assumed to be legal effects, so the set of effects is a convex subset of the dual vector space $V$. A measurement with $M$ outcomes is then a set of effects $\left\{f_{j}\right\}_{j=1}^{M}$ summing to the unit effect $e=\sum_{j=1}^{M} f_{j}$. This ensures that outcome probabilities of measurements sum to one. It is convenient to introduce the cone generated by the zero effect, the unit effect, and all other effects, i.e., the set $V^{+} \equiv\{t f \mid t \geq 0, f$ is an effect $\}$.

The triple $\left(V, V^{+}, e\right)$ is known as an ordered unit vector space and encodes all of the theoretically possible local effects of a GPT. Throughout the following we regard ( $V$, $\left.V^{+}, e\right)$ as the fundamental defining representation of a GPT with state space as a derived concept (i.e., $\Omega$ is henceforth defined as the set of all positive linear functionals $\omega$ on $V$ such that $e(\omega)=1)$. It is convenient to assume a further property, namely, that the triple $\left(V, V^{+}, e\right)$ is Archimedean.
This means that if $t e+f \in V^{+}$for all $t>0$, then $f \in V^{+}$. Such Archimedean ordered unit vector spaces are referred to as AOU spaces in the sequel. The Archimedean axiom is a kind of closure assumption which allows us, for example, to construct the order norm $\|f\|_{+} \equiv \inf \{t \mid t e \pm$ $\left.f \in V^{+}, t \geq 0\right\}$. All ordered vector spaces can be Archimedeanized [25], and from now on we assume that the effects of a GPT are suitably represented by an AOU space.

An important example of a GPT is that of quantum mechanics itself: an $n$-level quantum system is described by an AOU space where $V \subset M_{n}(\mathbb{C})$ is the set of $n \times n$ Hermitian matrices. The effects are then the matrices $F \in$ $V$ with $0 \leq F \leq \mathbb{1}$ and the unit is $e \equiv \mathbb{1}$. The cone $V^{+}$ generated by these effects is hence given by the positive semidefinite matrices. One can verify that the triple $\left(V, V^{+}, e\right)$ is Archimedean. State space $\Omega$ is given by $\left\{F \mapsto \operatorname{tr}(\rho F) \mid \rho \in V^{+}, \operatorname{tr}(\rho)=1\right\}$ and the order norm $\|A\|_{+}$is given by the largest singular value of $A$.

Sections of GPTs.-Here we study the effective theories arising from GPTs when only a subset of the possible effects may be implemented. For this purpose it is useful to introduce the notion of a linear map between AOU spaces: we say that a linear map $\varphi: V \rightarrow W$ between two AOU spaces $\left(V, V^{+}, e_{V}\right)$ and $\left(W, W^{+}, e_{W}\right)$ is positive if $\varphi\left(V^{+}\right) \subset W^{+}$and $\varphi$ is unital when $\varphi\left(e_{V}\right)=e_{W}$.

Our definition of a section of a GPT/AOU space $W$ is then motivated by the observation that if we can only implement some subset of the effects in $W^{+}$then we can implement any convex combination of them. A particular example of such a restriction is the intersection of $W^{+}$ with some subspace $V \subset W$. Since we can always apply the "do nothing" measurement, we require the subspace $V$ to contain $e_{W}$. Abstractly, a section of $\left(W, W^{+}, e_{W}\right)$ is defined to be a positive unital injection $\phi: V \hookrightarrow W$ such that $\phi\left(V^{+}\right)=W^{+} \cap \operatorname{im} \phi$. This condition has the consequence that the left inverse $\phi^{-1}$ is also a positive unital linear map.

When restricted to a section of a GPT $\left(W, W^{+}, e_{W}\right)$ the state space of the section $\left(V, V^{+}, e_{V}\right)$ is given by a quotient of the state space of $W$, i.e., $\Omega_{V}=\Omega_{W} / \sim$, where the equivalence relation is determined by $\omega \sim \sigma$ if $f(\omega)=$ $f(\sigma)$ for all $f \in V$. This quotient is the shadow of the convex body $\Omega_{W}$ on the subspace $V$.

We now describe the AOU space playing the central role in our argument. This space is given by triple $\left(\mathbb{R}^{n+1}\right.$, $\left.C_{n+1}^{+}(c),(1, \overrightarrow{0})\right)$ where $C_{n+1}^{+}(c)$ denotes the $(n+1)$ dimensional Euclidean cone with length-diameter ratio $c: 2$, i.e.,

$$
C_{n+1}^{+}(c)=\left\{(t, \vec{x}) \in \mathbb{R}_{+} \times \mathbb{R}^{n} \mid t \geq c\|\vec{x}\|_{2}\right\},
$$

of which $e=(1, \overrightarrow{0})$ is the order unit.

It is a nontrivial fact that this space can be embedded into a quantum system; i.e., it is a section of QM. The argument is due to Tsirelson [20] and proceeds as follows. 
Let $m=n / 2$ if $n$ is even and $m=(n+1) / 2$ for odd $n$ and define $\gamma_{1}, \ldots, \gamma_{2 m} \in M_{2^{m}}(\mathbb{C})$ via $\gamma_{2 j-1}=$ $\sigma_{z}^{(1)} \cdots \sigma_{z}^{(j-1)} \sigma_{x}^{(j)}$ and $\gamma_{2 j}=\sigma_{z}^{(1)} \cdots \sigma_{z}^{(j-1)} \sigma_{y}^{(j)}$, where we've employed the standard Pauli matrix notation and juxtaposition indicates an implicit tensor product. Consider the positive unital injection

$$
\varphi:(t, \vec{x}) \mapsto t \mathbb{1}+c \sum_{j} x_{j} \gamma_{j} .
$$

[The positivity follows from $2 t \varphi(t, \vec{x})=\varphi(t, \vec{x})^{2}+\left(t^{2}-\right.$ $\left.c^{2}\|x\|_{2}^{2}\right) \mathbb{1} \geq 0$, arising from $\gamma_{j} \gamma_{k}+\gamma_{k} \gamma_{j}=2 \delta_{j k} \mathbb{1}$.] Since $\varphi$ is an injection, it has a left-inverse

$$
\varphi^{\prime}: A \mapsto\left[\operatorname{tr} A, \operatorname{tr}\left(A \gamma_{i}\right) / c\right] / 2^{m},
$$

which is again positive. (Let $x_{i} \equiv \operatorname{tr}\left(A \gamma_{i}\right)$, so that $\operatorname{tr}(A)-$ $c\|\vec{x} / c\|_{2}=\operatorname{tr}\left[A \varphi\left(1,-\left(\vec{x} /\|\vec{x}\|_{2}\right) / c\right)\right] \geq 0$, since both matrices in the trace are already positive.)

Multipartite systems.-We now discuss how to form joint systems in the GPT framework. Suppose Alice and Bob are each in possession of a GPT $\left(V_{A}, V_{A}^{+}, e_{A}\right)$ and $\left(V_{B}, V_{B}^{+}, e_{B}\right)$, respectively, which describes the purely local measurements for each party. The joint GPT is then defined to be the AOU space $\left(V_{A} \otimes V_{B}, V_{A B}^{+}, e_{A} \otimes e_{B}\right)$ where, in order to proceed, we must specify how to construct the cone $V_{A B}^{+} \equiv$ " $\left(V_{A} \otimes V_{B}\right)^{+}$". There is an infinite variety of possibilities; however, we may restrict our attention to the following two extremal definitions [26]. The first corresponds to the maximal tensor product $\left(V_{A} \otimes_{\max } V_{B}\right)^{+}$which is defined to be the Archimedeanization of the cone $\left\{\sum_{j=1}^{k} f_{j} \otimes g_{j} \mid f_{j} \in\right.$ $\left.V_{A}^{+}, g_{j} \in V_{B}^{+}, k \in \mathbb{N}\right\}$ and the second to the minimal tensor product $\left(V_{A} \otimes_{\min } V_{B}\right)^{+} \equiv\left\{u \in V_{A} \otimes V_{B} \mid\left(\omega_{A} \otimes \omega_{B}\right)\right.$ $(u) \geq 0$, for all $\omega_{A} \in \Omega_{A}$ and $\omega_{B} \in \Omega_{B}$.

By way of contrast, the tensor product used in the formation of joint systems in quantum mechanics is neither the minimal nor maximal one, but is rather strictly in between: $\left(V_{A} \otimes_{\max } V_{B}\right)^{+} \subset\left(V_{A} \otimes_{\mathrm{QM}} V_{B}\right)^{+} \subset\left(V_{A} \otimes_{\min } V_{B}\right)^{+}$. The quantum mechanical tensor cone $V_{A B}^{+}$is given by the set of positive semidefinite operators in $M_{n_{A}}(\mathbb{C}) \otimes M_{n_{B}}(\mathbb{C})$. The state space $\Omega_{A B}^{\min }$ corresponding to $\left(V_{A} \otimes_{\min } V_{B}\right)^{+}$is precisely the set of separable states and the state space $\Omega_{A B}^{\max }$ corresponding to $\left(V_{A} \otimes_{\max } V_{B}\right)^{+}$is given by the set of all positive semidefinite operators $W$ with $\operatorname{tr}(W)=1$ which satisfy $\operatorname{tr}(W A \otimes B) \geq 0, \forall A, B \geq 0$. This set is dual to the set of entanglement witnesses [27] and includes all legal density operators as well as some operators with negative eigenvalues. Even though the state space $\Omega_{A B}^{\max }$ in the case where our local GPTs are QM is strictly larger than quantum mechanical state space, results of Refs. [21,22] show that it does not give rise to any bipartite correlations going beyond QM. The following proposition is a slight generalization of this statement, dealing with (local) sections of quantum systems.

Proposition 1.-Consider two AOU spaces $\left(V_{A}, V_{A}^{+}, e_{A}\right)$ and $\left(V_{B}, V_{B}^{+}, e_{B}\right)$ which are sections of quantum systems with according positive unital injections $\varphi_{A}$ and $\varphi_{B}$ into an
$n_{A}$-level (respectively, $n_{B}$-level) quantum system. Assume, without loss of generality, that $n_{A} \leq n_{B}$. Then for any positive unital bilinear map $\omega_{A B}: V_{A} \times V_{B} \rightarrow \mathbb{R}$ there exists a state $\sigma_{A B}$ of the composite quantum system $A B$ and a positive unital automorphism $\psi$ on $B$ such that $\omega_{A B}(f, g)=\operatorname{tr}\left[\sigma_{A B} \varphi_{A}(f) \otimes\left(\psi \circ \varphi_{B}\right)(g)\right]$.

Proof.-By assumption the map $\omega_{A B}^{\prime}\left(M_{A}, M_{B}\right) \mapsto$ $\omega_{A B}\left[\varphi_{A}^{-1}\left(M_{A}\right), \varphi_{B}^{-1}\left(M_{B}\right)\right]$ is positive and unital on the quantum systems $A, B$. Hence the statement reduces to the case where $\varphi_{A}$ and $\varphi_{B}$ are both the identity mapping. A proof for this case was given by Barnum et al. [21].

We stress that the existence of positive unital left inverse maps $\varphi_{A}^{-1}$ and $\varphi_{B}^{-1}$ is essential for this result to hold. Indeed, in the case of a hypothetical nonlocal box [28], it is impossible to find positive unital maps into QM so that their left inverse is also positive. Hence nonlocal boxes allow postquantum behavior. It is also important to note that Proposition 1 does not generalize to more than two parties [22].

Typical sections, main result.-Consider an arbitrary pair of $n$-dimensional GPTs $A$ and $B$ and suppose that we are only able to access a typical section of the set of local effects for $A$ (respectively, $B$ ). This is modeled by the intersection of $V_{A}^{+}$(respectively, $V_{B}^{+}$) with a typical $k$-dimensional subspace, $k \ll n$. To do this abstractly we choose a bijection $T$ between $V$ and $\mathbb{R}^{n}$ and consider a random linear injection $X: \mathbb{R}^{k} \hookrightarrow \mathbb{R}^{n}$ such that the random variable $X(\vec{x})$ is distributed according to the uniform measure on the Euclidean $(n-1)$-sphere of radius $\|\vec{x}\|_{2}$. (That is, $X$ is an $O(n)$-random rotation of an embedded fiducial $k$-dimensional subspace.) We call

$$
Q(t, \vec{x})=t e+T X(\vec{x})
$$

a centered random section of $\mathbb{R}^{k+1}$ into $V$ and it ensures that every subspace corresponding to a typical choice of measurement settings contains the neutral effect $e$. Since only convex combinations of $e$ with $T X\left(\mathbb{R}^{k}\right)$ are feasible, we now study the cone $V^{+} \cap Q\left(\mathbb{R}_{+}, \mathbb{R}^{k}\right)$.

The following result captures the concentration-ofmeasure phenomenon for our setting.

Proposition 2.-Let $\left(V, V^{+}, e\right)$ be an $n$-dimensional AOU space and $0<\varepsilon<1$. Then for $k \leq \mathcal{O}\left(\varepsilon^{2} \log n\right)$ there exists a $k+1$ dimensional centered random section $Q$ of $V$, such that, with high probability,

$$
Q\left[C_{k+1}^{+}(1+\varepsilon)\right] \subset V^{+} \cap Q\left(\mathbb{R}_{+}, \mathbb{R}^{k}\right) \subset Q\left[C_{k+1}^{+}(1-\varepsilon)\right] .
$$

Proof.-At the heart of the proof is the following "tangible" version of Dvoretzky's theorem [14,17,29]: If $\eta: S^{n-1} \rightarrow \mathbb{R}$ is a Lipschitz function with constant $L$ and central value 1 (with respect to the uniform spherical measure on $S^{n-1}$ ), then for every $\varepsilon>0$, if $E \subset \mathbb{R}^{n}$ is a random subspace of dimension $k \leq k_{0}=c_{0} \varepsilon^{2} n / L^{2}$, we have, that 


$$
\operatorname{Prob}\left[\sup _{S^{n-1} \cap E}|\eta(\vec{x})-1|>\varepsilon\right] \leq c_{1} e^{-c_{2} k_{0}},
$$

where $c_{0}, c_{1}$, and $c_{2}$ are absolute constants.

For our scenario, we use $\eta(\vec{z})=\inf \{t>0 \mid t e+T \vec{z} \in$ $\left.V^{+}\right\}$with $T$ chosen such that $\eta$ has a mean (which is a particular central value) of 1 on the $(n-1)$-dimensional Euclidean sphere and that the Lipschitz constant $L$ of $\eta$ is bounded via $L \leq c^{\prime} \sqrt{n / \log n}$ for some absolute constant $c^{\prime}$. This is always possible, as can be seen following the proof of Theorem 4.3 in Ref. [29]: First, by a lemma of Dvoretzky and Rogers, cf. Theorem 3.4 in Ref. [14], the bijection $T$ can be chosen such that for all canonical vectors $\vec{e}_{k}$ with $k \leq$ $n / 2$ it holds that $\left\|T \vec{e}_{k}\right\|_{+} \geq\|T\| / 4$. Without loss of generality we may assume in addition that $\eta$ has mean 1 . Then, for a vector of normal distributed variables $\vec{g}$ and due to $\|T \vec{z}\|_{+}=\max \{\eta(\vec{z}), \eta(-\vec{z})\}$ and due to Eqs. (4.14) and (4.18) in Ref. [29] we find,

$$
\begin{aligned}
2 \sqrt{n} & \geq 2 \mathbb{E} \eta(\vec{g}) \geq \mathbb{E}\|T \vec{g}\|_{+} \geq \mathbb{E} \max _{k}\left|g_{k}\right|\left\|T \vec{e}_{k}\right\|_{+} \\
& \geq \mathbb{E} \max _{k \leq n / 2}\left|g_{k}\right|\left\|T \vec{e}_{k}\right\|_{+} \geq c^{\prime \prime} \sqrt{\log (n / 2)}\|T\| / 4 .
\end{aligned}
$$

On the other hand, $\eta$ is a sublinear function and thus

$$
\begin{gathered}
\left|\eta\left(\vec{z}_{1}\right)-\eta\left(\vec{z}_{2}\right)\right| \leq \max \left\{\eta\left(\vec{z}_{1}-\vec{z}_{2}\right), \eta\left(\vec{z}_{2}-\vec{z}_{1}\right)\right\} \\
=\left\|T\left(\vec{z}_{1}-\vec{z}_{2}\right)\right\|_{+} \leq\|T\|\left\|\vec{z}_{1}-\vec{z}_{2}\right\|_{2},
\end{gathered}
$$

which eventually shows $L \leq c^{\prime} \sqrt{n / \log n}$.

Now, by virtue of Dvoretzky's theorem, the following holds with high probability. For all $\vec{x} \neq 0$ with $\xi \equiv\|\vec{x}\|_{2} \leq$ $1 /(1+\varepsilon)$, we have $\eta[X(\vec{x} / \xi)] \leq 1+\varepsilon \leq 1 / \xi$, and hence $Q(1, \vec{x})=[e / \xi+T X(\vec{x} / \xi)] \xi \in V^{+}$. Conversely, for all $\vec{x}$ with $\xi \equiv\|\vec{x}\|_{2}>1 /(1-\varepsilon)$, we have $\eta[X(\vec{x} / \xi)] \geq$ $1-\varepsilon>1 / \xi$, i.e., $Q(1, \vec{x}) \notin V^{+}$. The converse statement completes the proof.

Thus, with high accuracy, the effective theory corresponding to a low-dimensional $O(n)$-typical section of a local GPT looks like a Euclidean AOU space, cf. Fig. 1 for an illustration. The cones $Q\left(C_{k+1}^{+}(1 \pm \varepsilon)\right)$ give a very accurate description of the typical section, since by linearity all observable probabilities may at most deviate by $\mathcal{O}(\varepsilon)$. Combining this with our previous finding, namely that Euclidean cones are sections of QM, and hence, in view of Proposition 1, all bipartite correlations of their maximal tensor product may be simulated within QM, we arrive at our anticipated main result. Conversely, due to an argument by Tsirelson [20], all bipartite dichotomic correlations can be explained within a Euclidean cone of appropriate dimension. Our result reduces to this dichotomic case, since already our description of a GPT by an AOU space is essentially limited to the dichotomic case.

Finally we briefly discuss the situation of a generalized Popescu-Rohrlich (PR) box, which exhibits (in some

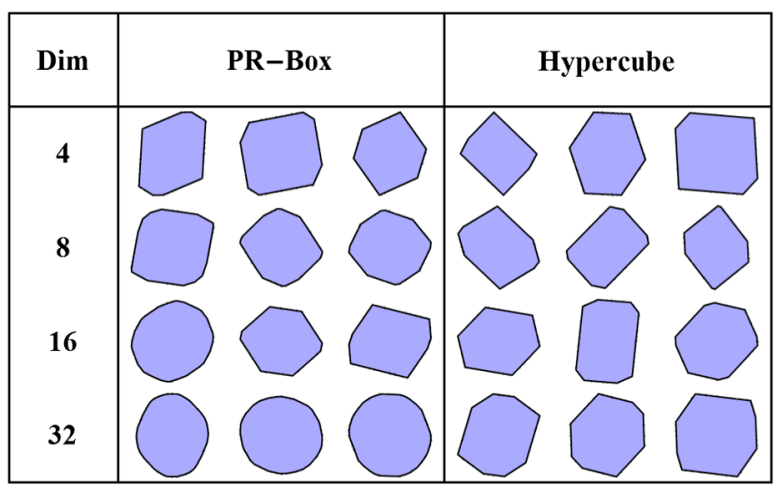

FIG. 1 (color online). Typical two-dimensional sections of a hypercube and of the effect space of a PR box in various dimensions. In both cases an increasing rounding of the corners of the sections can be observed. However in the case of a hypercube, which is the extremal situation for Dvoretzky's theorem, there is still an appreciable probability for nonrounded sections, due to low dimensionality.

sense) the "maximal" possible postquantum correlations [28]. Such boxes are locally described by an AOU vector space over $\mathbb{R}^{n}$ with cone $\mathrm{PR}^{+}=\left\{(t, \vec{x})\left|t \geq \sum_{i}\right| x_{i} \mid\right\}$ and neutral element $(1, \overrightarrow{0})$. By virtue of Proposition 2 , the fraction of three-dimensional sections from a $55 \times$ $10^{6}$-dimensional box with a postquantum behavior of more than $\pm 3 \%$ is as low as $10^{-6}$ [30].

Conclusions.-We have presented a mechanism whereby observable bipartite correlations of an arbitrary postquantum theory could be, with high accuracy, compatible with those exhibited by quantum mechanics. Our argument exploited the concentration-of-measure phenomenon and hence works for any typical low-dimensional section of a generalized probabilistic theory. We argued that such typical sections arise due to a lack of ultraprecise experimental control, in which case it would be virtually impossible to observe any postquantum behavior, even if the fundamental theory of nature wasn't quantum mechanics. This is complementary to the emergence of classicality from quantum mechanics via decoherence [8,9], since we consider only a pair of (microscopic) objects, rather than an ensemble of objects. Our argument indicates that there is another option for refinement of today's physics: we might be missing hidden postquantum structures due to ignorance of the correct measurement directions.

We thank O. Gühne, A. Ahlbrecht, and C. Budroni for helpful discussions and the Centro de Ciencias de Benasque, where part of this work was done, for its hospitality during the workshop on quantum information 2012. This work has been supported by the Austrian Science Fund (FWF): Y376-N16 (START prize), the BMBF (CHIST-ERA network QUASAR), the EU (Marie-Curie CIG 293933/ENFOQI, Coquit, QFTCMPS), V. B. S. is supported by an ETH postdoctoral fellowship and the SNF through the National Centre of Competence in 
Research Quantum Science and Technology." This work was supported, in part, by the cluster of excellence EXC 201 "Quantum Engineering and Space-Time Research," by the Deutsche Forschungsgemeinschaft (DFG).

*matthias.kleinmann@uni-siegen.de

†tobias.osborne@itp.uni-hannover.de

†scholz@phys.ethz.ch

§albert.werner@itp.uni-hannover.de

[1] H. Barnum, J. Barrett, M. Leifer, and A. Wilce, Phys. Rev. Lett. 99, 240501 (2007).

[2] H. Barnum and A. Wilce, Electron. Notes Theor. Comput. Sci. 270, 3 (2011).

[3] J. Barrett, Phys. Rev. A 75, 032304 (2007).

[4] L. Masanes and M.P. Müller, New J. Phys. 13, 063001 (2011).

[5] G. Chiribella, G. M. D'Ariano, and P. Perinotti, Phys. Rev. A 81, 062348 (2010).

[6] J. Barrett, L. Hardy, and A. Kent, Phys. Rev. Lett. 95, 010503 (2005).

[7] E. Hänggi, R. Renner, and S. Wolf, in EUROCRYPT (Springer, Berlin, 2010), pp. 216-234.

[8] M. Navascués and H. Wunderlich, Proc. R. Soc. A 466, 881 (2010).

[9] P. Kurzyński, A. Soeda, R. Ramanathan, A. Grudka, J. Thompson, and D. Kaszlikowski, arXiv:1111.2696v2.

[10] G. Ludwig, An Axiomatic Basis for Quantum Mechanics (Springer-Verlag, Berlin Heidelberg, 1987), Vol. I-II.

[11] P. Mittelstaedt, The Interpretation of Quantum Mechanics and the Measurement Process (Cambridge University Press, Cambridge, England, 1998).

[12] L. Hardy, arXiv:quant-ph/0101012v4.

[13] E. M. Alfsen and F. W. Shultz, Geometry of State Spaces of Operator Algebras (Springer, New York, 2003).

[14] V.D. Milman and G. Schechtman, Asymptotic Theory of Finite Dimensional Normed Spaces, Lecture Notes in Mathematics Vol. 1200 (Springer-Verlag, Berlin, 1986).

[15] M. Talagrand, Inst. Hautes Études Sci. Publ. Math. 81, 73 (1995).

[16] P. Hayden, D. W. Leung, and A. Winter, Commun. Math. Phys. 265, 95 (2006).

[17] G. Aubrun, S. Szarek, and E. Werner, Commun. Math. Phys. 305, 85 (2011).

[18] M. J. Bremner, C. Mora, and A. Winter, Phys. Rev. Lett. 102, 190502 (2009).

[19] D. Gross, S. T. Flammia, and J. Eisert, Phys. Rev. Lett. 102, 190501 (2009).

[20] B.S. Tsirel'son, Zap. Nauchn. Sem. Leningrad. Otdel. Mat. Inst. Steklov. (LOMI) 142, 174 (1985) [J. Sov. Math. 36, 557 (1987)].

[21] H. Barnum, S. Beigi, S. Boixo, M. B. Elliott, and S. Wehner, Phys. Rev. Lett. 104, 140401 (2010).

[22] A. Acín, R. Augusiak, D. Cavalcanti, C. Hadley, J. K. Korbicz, M. Lewenstein, L. Masanes, and M. Piani, Phys. Rev. Lett. 104, 140404 (2010).

[23] E. M. Alfsen, Compact Convex Sets and Boundary Integrals, Ergebnisse der Mathematik und ihrer Grenzgebiete Vol. 57 (Springer, Berlin, 1971).

[24] V. I. Paulsen, Completely Bounded Maps and Operator Algebras, Cambridge Studies in Advanced Mathematics Vol. 78 (Cambridge University Press, Cambridge, England, 2002).

[25] V. I. Paulsen and M. Tomforde, Indiana University mathematics Journal 58, 1319 (2009).

[26] K. H. Han, arXiv:0906.1858v1.

[27] M. Horodecki, P. Horodecki, and R. Horodecki, Phys. Lett. A 223, 1 (1996).

[28] S. Popescu and D. Rohrlich, Found. Phys. 24, 379 (1994).

[29] G. Pisier, The Volume of Convex Bodies and Banach Space Geometry, Cambridge Tracts in Mathematics Vol. 94 (Cambridge University Press, Cambridge, England, 1989).

[30] Those estimates stem from estimating the constants $c_{0}, c_{1}$, and $c_{2}$ in the original proofs. Numerically, the required dimensions is about 2000. 